\section{Observer la cellule et ses structures membranaires à l'échelle du nanomètre}

Amélie Leforestier, Nicolas Lemercier, Françoise Livolant
CNRS UMR 8502, laboratoire de physique des solides, bâtiment 510, Université Paris-Sud, 91405 Orsay Cedex, France.

amelie.leforestier@u-psud.fr
> L'imagerie cellulaire et tissulaire connaît depuis une dizaine d'années des progrès spectaculaires grâce à l'exploitation des propriétés de la fluorescence. Elle permet une imagerie en trois dimensions (3D), voire 4D, de la cellule vivante à des résolutions toujours améliorées [1-3], et permet même la détection et le suivi de molécules uniques. Aussi riches soientelles, ces approches ne disent rien sur la conformation des macromolécules, la nature de leurs interactions, ou leur organisation supramoléculaire ; d'où le besoin d'informations à plus haute résolution.

La microscopie électronique à transmission (MET) est l'outil idéal d'investigation, même si le vide poussé dans lequel se propagent les électrons ne permet pas encore d'explorer la structure de la cellule vivante. Depuis trente ans, la cryoMET s'est imposée comme un outil majeur en biologie structurale. Associée à des analyses d'images sophistiquées, elle permet d'obtenir des résolutions quasi atomiques de gros complexes macromoléculaires et de particules virales, isolés en solution [4]. Mais elle reste limitée à des objets de petite dimension (idéalement 50 à $100 \mathrm{~nm}$, au plus 500 à $700 \mathrm{~nm}$ ). Bien qu'apparue à la même époque, la cryo-MET de sections vitreuses (CEMOVIS pour cryo-electron microscopy of vitreous sections) est une technique encore émergente que seuls quelques laboratoires dans le monde développent à l'heure actuelle. Elle consiste à geler les cellules à très basse température $\left(\leqslant-196^{\circ} \mathrm{C}\right)$, suffisamment rapidement pour éviter la cristallisation de l'eau (vitrification), puis à réaliser à une température inférieure à la température de dévitrification de l'eau $\left(-135^{\circ} \mathrm{C}\right)$, des cryosections ultraminces (25 à $50 \mathrm{~nm}$ ) qui sont observées à $-180^{\circ} \mathrm{C}$ sous de faibles doses d'électrons. On obtient ainsi des ins-

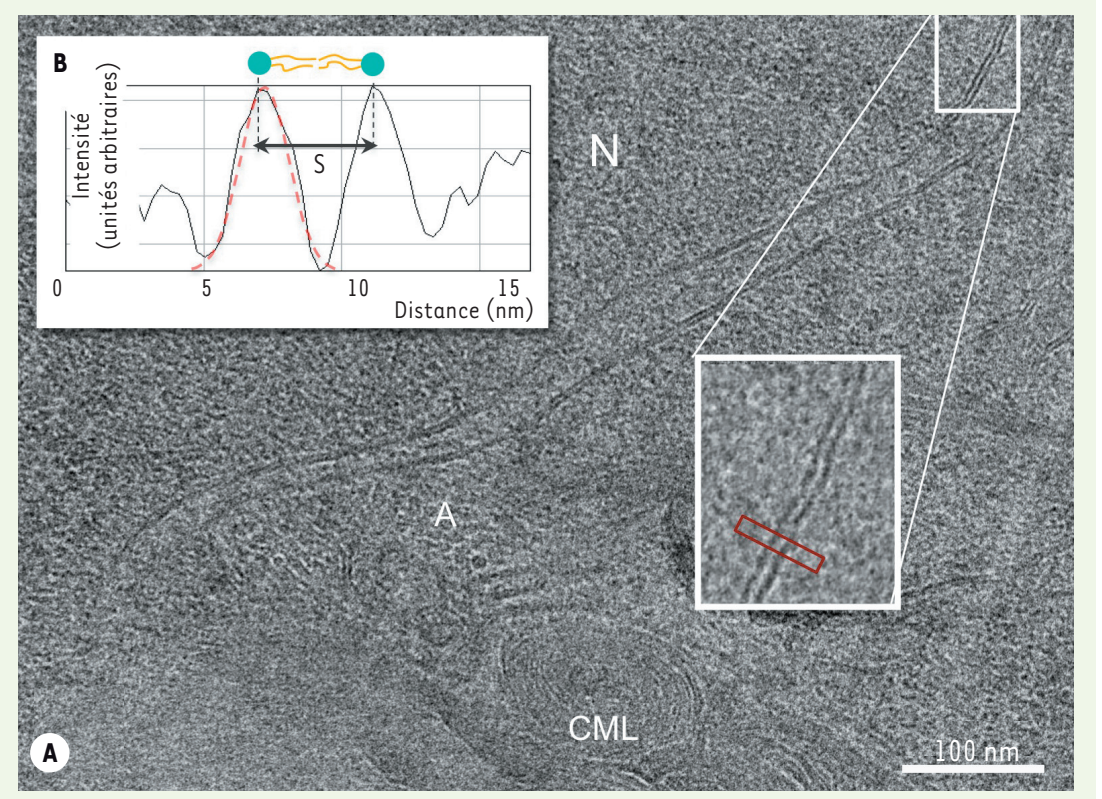

tantanés de la cellule dans son état natif, sans coloration ni marquage [6].

Nous allons montrer ici ce que peut apporter cette méthode à l'étude des systèmes membranaires à l'aide de quelques exemples puisés dans différents types cellulaires [7].

\section{Visualiser les bicouches de phospholipides}

Les membranes qui délimitent cellules et compartiments cellulaires sont facilement identifiables sur les images de CEMOVIS lorsqu'elles sont observées en section parfaitement transversales. En optimisant les conditions d'imagerie, les deux feuillets de phospholipides peuvent alors être mis en évidence : les membranes se présentent comme deux lignes parallèles, denses aux électrons, correspondant aux têtes polaires des phospholipides, et séparées par une ligne claire correspondant à leurs chaînes aliphatiques (Figure 1). Au-delà de cette structure en bicouche déjà bien connue, on peut ainsi analyser les dissymétries d'épaisseur et de composition des deux feuillets, et découvrir la présence de membranes finement accolées au sein de plusieurs

Figure 1. Visualisation des structures membranaires. A. Vue d'une partie d'une cellule de la lignée colique humaine HT29. Le noyau (N) est limité par l'enveloppe nucléaire. Des filaments d'actine $(A)$ et des corps multilamellaires (CML) sont visibles dans le cytoplasme. La structure de la membrane en double feuillet est bien visible. B. Le profil d'intensité, mesuré perpendiculairement à la membrane le long du rectangle rouge, permet de mesurer la distance (S) (ici 3,5 nm) séparant les deux rangées de têtes polaires des phospholipides. 


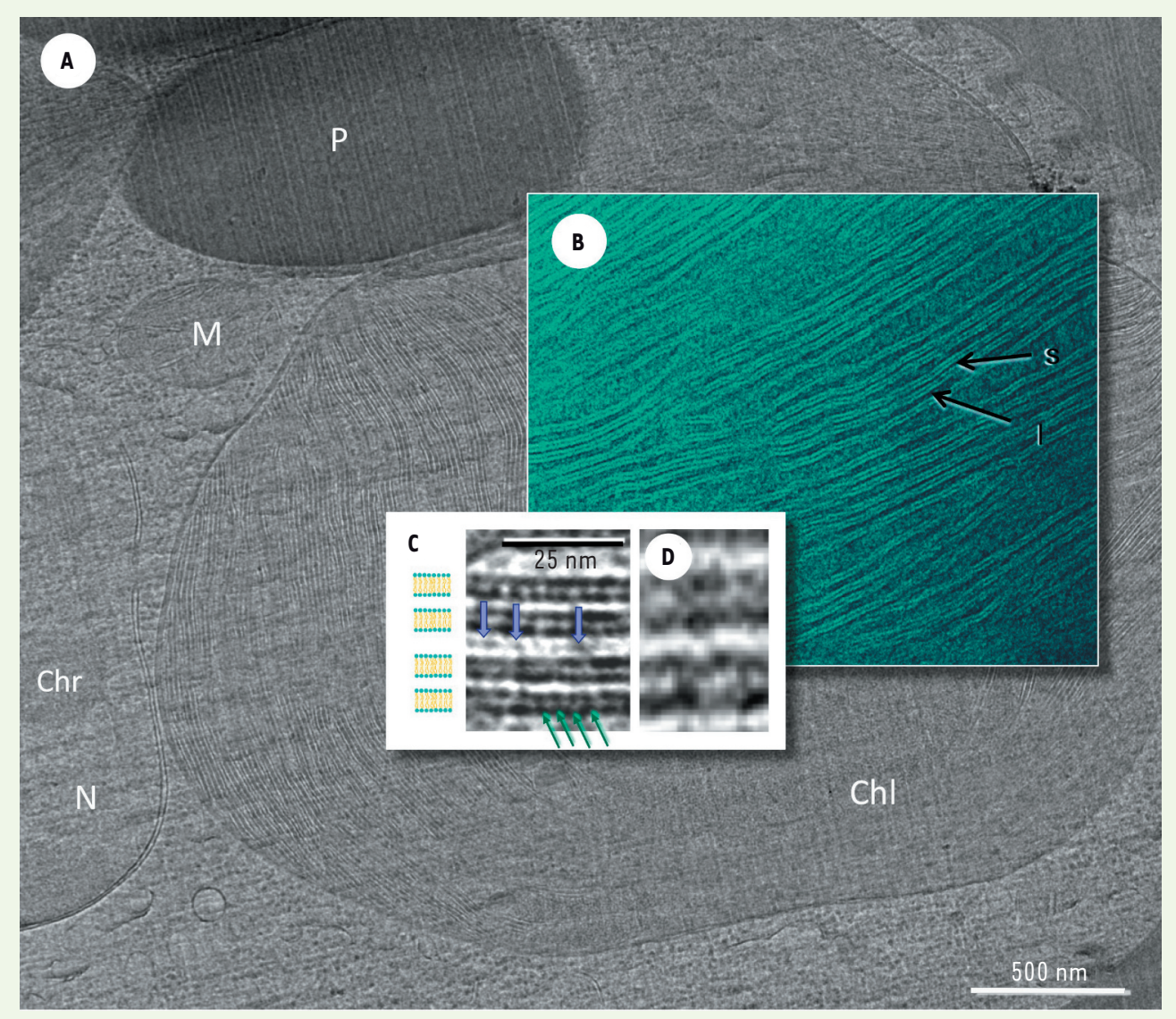

Figure 2. Réseaux de protéines transmembranaires.

$A$. Vue générale d'une algue unicellulaire (Euglena gracilis). On reconnaît le noyau (N) avec ses chromosomes (Chr), une mitochondrie (M), un granule de stockage ou paramylon $(P)$ et le chloroplaste (Chl). B. Détail d'un chloroplaste montrant l'empilement de thylakoïdes en vue transversale ; les régions luminales des thylakoïdes (I) alternent avec le stroma (S). C. Détail d'un empilement de thylakoïdes. Au sein des bicouches sont visibles des séries de protéines transmembranaires (photosystèmes I et II, flèches vertes). La lumière du thylakoïde (voir I sur panneau B) présente une densité correspondant au complexe protéique $0 \varepsilon C$

(oxygen evolving complex, flèches bleues). D. Par comparaison, un empilement de thylakoïdes d'épinard observé sur un cryotomogramme est présenté ici à la même échelle et permet d'obtenir directement des données 3D, mais avec une moindre résolution (reproduit de [13]).

types cellulaires (au niveau des réservoirs alvéolaires calciques de la paramécie ou des corps multilamellaires de lignées cellulaires humaines par exemple [7]). De tels contacts étroits n'avaient jamais été détectés par manque de résolution spatiale. Cependant, ces contacts pourraient être fréquents au sein des cellules eucaryotes, ce qui soulève des interrogations sur leurs implications biologiques et, plus particulièrement, sur les mécanismes de fusion mis en jeu au sein de la cellule.

\section{Analyser les réseaux de protéines transmembranaires}

Les membranes thylakoïdes ${ }^{1}$ photosynthétiques des chloroplastes fournissent

\footnotetext{
${ }^{1}$ Les chloroplastes ont deux membranes (trois dans le cas de l'algue Euglena gracilis) bordant une zone aqueuse appelée stroma. Ce dernier contient la membrane thylakoïde, plissée en un réseau de nombreuses vésicules aplaties, qui peuvent s'organiser en des empilements compacts.
}

un très bel exemple de réseau protéique fonctionnel ordonné à trois dimensions (Figure 2). La présence des protéines est détectée par une augmentation locale de l'intensité du signal dans la région des chaînes aliphatiques des phospholipides. La résolution atteinte permet d'analyser l'ordre entre protéines dans le plan des membranes aussi bien que dans le plan perpendiculaire aux empilements fonctionnels. La Figure $2 C$ montre un exemple des détails accessibles par imagerie CEMOVIS. Les séries de photosystèmes I et II (flèches vertes) sont visibles au sein des bicouches alors que la lumière du thylakoïde présente des densités correspondant au complexe protéique 0\&C (oxygen evolving complex, flèches mauves), deux composants de la chaîne respiratoire. Alors qu'on connaît aujourd'hui à l'échelle atomique les structures de chacun des principaux complexes protéiques impliqués dans les réactions photosynthétiques, ce type d'approche permet d'appréhender leur organisation spatiale et leurs interactions in situ.

L'imagerie cellulaire par CEMOVIS ouvre ainsi la voie à un niveau de résolution encore inexploré in situ, celle du nanomètre. Outre l'analyse des systèmes membranaires, cette méthode s'est déjà avérée particulièrement fructueuse pour l'étude de complexes macromoléculaires de grande taille (par exemple microtubules ou desmosomes [8]) mais aussi de systèmes fibrillaires, tels que I'ADN dans le noyau des spermatozoïdes [9], ou le nucléoïde bactérien. En couplant l'analyse d'images de cryosections (pour lesquelles les conditions d'obtention des images ont été optimisées et qui procurent ainsi des données haute résolution mais à 2D) et l'acquisition de données 
par cryo-tomographie $[10,11]$ (qui permet de s'abstraire des problèmes de superposition, mais au prix d'une limitation de la résolution à quelques $4-7 \mathrm{~nm}$ [Figure 2D]), on peut espérer accéder prochainement à l'imagerie de la cellule entière à une résolution nanométrique, voire subnanométrique. L'une comme l'autre de ces méthodes ont l'avantage de ne requérir la présence d'aucun contrastant ce qui constitue un atout incontestable, puisque c'est la structure native de l'ensemble des molécules qui est observée. Néanmoins, elles sont toutes deux confrontées aux difficultés d'identification des composants de la cellule, pourtant indispensable pour dépasser la simple description structurale. Différentes voies de marquages possibles commencent à être envisagées (marqueurs clonables tels que la métal- lothionéine, pouvant lier des éléments lourds denses aux électrons [12]) qui s'avèreront essentielles dans les années qui viennent pour que la méthode s'impose comme majeure dans le paysage de la biologie intégrative. $\diamond$

Imaging the cell and its membranes at the nanometer scale

\section{LIENS D'INTÉRÊT}

Les auteurs déclarent n'avoir aucun lien d'intérêt concernant les données publiées dans cet article.

\section{RÉFÉRENCES}

1. Trugnan $G$, Fontanges $P$, Delautier D, Ait-Slimane T. FRAP, FLIP, FRET, BRET, FLIM, PRIM De nouvelles techniques pour voir la vie en couleur! Med Sci (Paris) $2004 ; 20: 1027-34$.

2. Débarre D, Pena AM, Supatto W, et al. Microscopies multi-harmoniques pour l'imagerie structurale de tissus intacts. Med Sci (Paris) 2006 ; $22: 845-50$.

3. Girard PP, Forget BC. Microscopie de fluorescence à feuille de lumière. Med Sci (Paris) 2011 ; 27 : 753-62.

4. Chiu W, Baker ML, Almo SC. Structural biology of cellular machines. Trends Cell Biol 2006; $16: 144-50$.
5. Zhou ZH. Atomic reolution cryo electron microscopy of macromolecular complexes. Adv Protein Chem Struct Biol $2011 ; 82$ : 1-35.

6. Al-Amoudi A, Chang JJ, Leforestier A, et al. Cryoelectron microscopy of vitreous sections. EMBO J $2004 ; 23: 3583-8$.

7. Leforestier A, Lemercier N, Livolant F. Contribution of cryoelectron microscopy of vitreous sections to the understanding of biological membrane structure. Proc Natl Acad Sci USA 2012 ; 109 : 8959-64.

8. Al-Amoudi A, Díez DC, Betts MJ, Frangakis AS. The molecular architecture of cadherins in native epidermal desmosomes. Nature 2007 ; 450 : 832-7.

9. Sartori Blanc N, Senn A, Leforestier A, et al. DNA in human and stallion spermatozoa forms local hexagonal packing with twist and many defects.) Struct Biol $2001 ; 134: 76-81$.

10. Yahav T, Maimon T, Grosmann $\varepsilon$, et al. Cryo-electron tomography: gaining insight into cellular processes by structural approaches. Curr Opin Struct Biol 2011 ; $21: 670-7$.

11. Hoenger A, Bouchet-Marquis C. Cellular tomography. Adv Protein Chem Struct Biol 2011 ; 62 : 2393-402.

12. Mercogliano CP, DeRosier DJ. Gold nanocluster formation using metallothionein: mass spectrometry and electron microscopy. J Mol Biol 2006 ; 355 : 211-23.

13. Daum B, Nicastro D, Austin J, et al. Arrangement of photosystem II and ATP synthase in chloroplast membranes of spinach and pea. Plant Cell 2010 ; 22 : 1299-1312.

NOUVELLE

Le switch angiogénique ou
comment réveiller les cellules
tumorales dormantes
Julie Bensimon

Rôle du switch angiogénique dans la sortie de dormance tumorale Le terme de «dormance tumorale » désigne la persistance de cellules tumorales en petit nombre dans l'organisme, sans croissance tumorale. Le phénomène de dormance tumorale est décrit depuis près d'un demi-siècle, mais les mécanismes le sous-tendant sont encore mal connus. Par exemple, en 1987, des autopsies médico-légales réalisées sur 110 femmes jeunes et d'âge moyen, décédées de causes non naturelles, ont montré, chez $20 \%$ d'entre elles, la présence de tumeurs microscopiques, alors qu'un cancer du sein n'avait été diagnostiqué que chez $1 \%$ d'entre elles [1].
Ces foyers tumoraux dormants peuvent non seulement précéder le développement d'un cancer in situ, mais, lorsque la tumeur primaire est détectable et diagnostiquée, ils peuvent également être à l'origine de micrométastases. Dans ce cas, la sortie de dormance détermine la prolifération métastatique du cancer dans l'organisme [2].

L'obtention de modèles expérimentaux de la dormance tumorale nécessite d'établir un équilibre entre l'hôte et la tumeur, comme le montre un modèle murin de tumeurs chimio-induites décrit par B. Quesnel dans médecine/sciences [3]. Dans ce modèle, certaines tumeurs induites restent dormantes jusqu'à ce
Laboratoire de cancérologie expérimentale (DSV/IRCM/SREIT/LCE),

18 , route du Panorama, $B P n^{\circ} 6$, 92265 Fontenay-aux-Roses Cedex, France. julie.bensimon86@gmail.com

que l'immunité adaptative de l'animal soit altérée, ce qui déclenche le développement des tumeurs [3]. Ce type de modèle met en évidence le rôle important joué par le système immunitaire de I'hôte dans le maintien des tumeurs à l'état de dormance : les tumeurs qui se développent sont celles qui parviennent à échapper au contrôle immunitaire. Un second processus clé dans la sortie de dormance est la capacité de la tumeur à contrôler l'angiogenèse. Ainsi, J. Folkman et D. Hanahan ont attribué en 1989 un rôle critique au «switch angiogénique » dans la sortie de dormance tumorale, c'est-à-dire à la capacité de la tumeur à établir un réseau vasculaire 